Pacific Journal of Mathematics

INTERPOLATION, CONTINUATION, AND QUADRATIC 


\title{
INTERPOLATION, CONTINUATION, AND QUADRATIC INEQUALITIES
}

\author{
W. Hengartner and G. Schober
}

1. C. H. FitzGerald considered conditions for interpolating the values of an analytic function in terms of quadratic inequalities. In addition, he used them to obtain an interesting result concerning analytic continuation of functions of two variables and related them to Pick-Nevanlinna interpolation.

We shall show that these theorems follow directly from well-known principles of functional analysis. Since these principles are not limited to interpolating values of an analytic function, we shall obtain applications to interpolating functional values of both analytic and harmonic functions of several variables. In addition, we obtain analogous applications to analytic and harmonic continuation and to Pick-Nevanlinna interpolation.

2. Abstract framework. Let $X$ be a reflexive topological vector space over the real or complex field and $X^{*}$ its topological dual space. Assume that $p\left(x^{*}\right)$ is a continuous semi-norm defined on $X^{*}$. A classical theorem of Helly and Hahn (see G. Köthe $[4, p$. 113]) is the following. Since the proof is brief, we include it for completeness.

THEOREM 2.1 (Helly-Hahn). Let $\left\{c_{i}\right\}_{i \in I}$ be a set of scalars and $\left\{x_{i}^{*}\right\}_{i \in I}$ a corresponding set of functionals in $X^{*}$. Then there exists an $x \in X$ such that

$$
\begin{aligned}
& \text { (i) } x_{i}^{*}(x)=c_{i} \text { for all } i \in I \text {, and } \\
& \text { (ii) }\left|x^{*}(x)\right| \leqq p\left(x^{*}\right) \text { for all } x^{*} \in X^{*} \text {, }
\end{aligned}
$$

if and only if

$$
\left|\sum_{k=1}^{N} \alpha_{i_{k}} c_{i_{k}}\right| \leqq p\left(\sum_{k=1}^{N} \alpha_{i_{k}} x_{i_{k}}^{*}\right)
$$

for every finite collection of scalars $\alpha_{i_{1}}, \cdots, \alpha_{i_{N}}$.

Proof. The necessity is trivial. For the sufficiency, let $A$ be the linear span of $\left\{x_{i}^{*}\right\}_{i \in I}$. Define a linear functional $x$ on $A$ by setting $x\left(\sum_{k=1}^{N} \alpha_{i_{k}} x_{i_{k}}^{*}\right)=\sum_{k=1}^{N} \alpha_{i_{k}} c_{i_{k}}$. Then $x$ is well defined since if $\sum \alpha_{i_{k}} x_{i_{k}}^{*}=\sum \beta_{i_{\kappa}} x_{i_{\kappa}}^{*}$, then (1) implies

$$
\left|\sum \alpha_{i_{k}} c_{i_{k}}-\sum \beta_{i_{\kappa}} c_{i_{\kappa}}\right| \leqq p\left(\sum \alpha_{i_{k}} x_{i_{k}}^{*}-\sum \beta_{i_{\kappa}} x_{i_{\kappa}}^{*}\right)=p(0)=0 .
$$


Obviously,

$$
\left|x\left(x^{*}\right)\right| \leqq p\left(x^{*}\right)
$$

for each $x^{*} \in A$. By the Hahn-Banach theorem we may extend $x$ to all of $X^{*}$ and (2) persists. Since $X$ is reflexive, we may identify $x$ with a point of $X$.

We shall be concerned with applications of the Helly-Hahn theorem. One important case occurs when the semi-norm $p$ is generated by a positive, continuous, conjugate-linear operator $T: X^{*} \rightarrow X$. Positive means that $x^{*}\left(T x^{*}\right) \geqq 0$ for every $x^{*} \in X^{*}$, and conjugate-linear means that $T\left(\alpha x^{*}\right)=\bar{\alpha} T x^{*}$ when the scalar field is complex. In this case $p\left(x^{*}\right)=\sqrt{x^{*}\left(T x^{*}\right)}$ is a continuous semi-norm on $X^{*}$ and the Helly-Hahn theorem takes the following form.

CoROLlaRY 2.1. Let $\left\{c_{i}\right\}_{i \in I}$ be a set of scalars and $\left\{x_{i}^{*}\right\}_{i \in I}$ a corresponding set of functionals in $X^{*}$. Suppose that $T: X^{*} \rightarrow X$ is a positive, continuous, conjugate-linear operator. Then there exists an $x \in X$ such that

(i) $x_{i}^{*}(x)=c_{i}$ for all $i \in I$ and

(ii) $\left|x^{*}(x)\right|^{2} \leqq x^{*}\left(T x^{*}\right)$ for all $x^{*} \in X^{*}$

if and only if

$$
\left|\sum_{k=1}^{N} \alpha_{i_{k}} c_{i_{k}}\right|^{2} \leqq \sum_{k, \kappa=1}^{N} \alpha_{i_{k}} \bar{\alpha}_{i_{\kappa}} x_{i_{k}}^{*}\left(T x_{i_{\kappa}}^{*}\right)
$$

for every finite collection of scalars $\alpha_{i_{1}}, \cdots, \alpha_{i_{N}}$.

A second case occurs when the semi-norm $p$ is generated by a bounded operator $T$ defined on a linear subspace of $X^{*}$ to $X$.

COROLLARY 2.2. Let $\left\{c_{i}\right\}_{i \in I}$ be a set of scalars and $\left\{x_{i}^{*}\right\}_{i \in I} a$ corresponding set of functionals in $X^{*}$. Suppose that $T$ is a bounded, conjugate-linear operator from the linear span $A$ of $\left\{x_{i}^{*}\right\}_{i \in I}$ to $X$. There exists an $x \in X$ such that

(i) $x_{i}^{*}(x)=c_{i}$ for all $i \in I$,

(ii) $\left|x^{*}(x)\right|^{2} \leqq x^{*}\left(T x^{*}\right)$ for all $x^{*}$ in the closure of $A$, and

(iii) $\|x\|^{2} \leqq\|T\|_{A}$

if and only if

$$
\left|\sum_{k=1}^{N} \alpha_{i_{k}} c_{i_{k}}\right|^{2} \leqq \sum_{k, \kappa=1}^{N} \alpha_{i_{k}} \bar{\alpha}_{i_{k}} x_{i_{k}}^{*}\left(T x_{i_{k}}^{*}\right)
$$

for every finite collection of scalars $\alpha_{i_{1}}, \cdots, \alpha_{i_{N}}$. 
Proof. The function $p\left(x^{*}\right)=\sqrt{\|T\|_{A}}\left\|x^{*}\right\|$ defines a (semi-)norm on $X^{*}$. Since $y^{*}=\sum_{k=1}^{N} \alpha_{i_{k}} x_{i_{k}}^{*}$ is in $A$, the condition (4) implies

$$
\left|\sum_{k=1}^{N} \alpha_{i_{k}} c_{i_{k}}\right|^{2} \leqq\left|y^{*}\left(T y^{*}\right)\right| \leqq\|T\|_{A}\left\|y^{*}\right\|^{2}=\left[p\left(y^{*}\right)\right]^{2}
$$

so that (1) holds. Now by the Helly-Hahn theorem an $x \in X$ exists such that (i) holds, and (iii) follows from the inequality

$$
\left|x\left(x^{*}\right)\right|=\left|x^{*}(x)\right| \leqq p\left(x^{*}\right)=V\|T\|_{A}\left\|x^{*}\right\| .
$$

Finally, (4) implies that $\left|x^{*}(x)\right|^{2} \leqq x^{*}\left(T x^{*}\right)$ for $x^{*} \in A$, and by continuity for all $x^{*}$ in the closure of $A$. The converse is trivial since (4) follows from (i) and (ii).

REMARKS. It is evident that $x$ is unique whenever $\left\{x_{i}^{*}\right\}_{i \in I}$ is a complete set in $X^{*}$ (i.e., the closure of $A$ is $X^{*}$ ).

Positive, continuous, conjugate-linear operators $T: X^{*} \rightarrow X$ occur naturally. For example, if $X$ is a Hilbert space, the familiar identification of $X^{*}$ with $X$ has these properties. See also $\S 5$.

3. An example. As an application of Corollary 2.2, we shall obtain the following result, and then note in the next section that a similar proof implies several significant generalizations.

THEOREM 3.1 (FitzGerald [2, Thm. 1.1]). Let $\left\{z_{n}\right\}_{n=1}^{\infty}$ be a sequence of distinct points in a domain $D$ of $C$, with an accumulation point in $D$. Suppose $K(z, \zeta)$ is defined on $D \times D$ and is analytic in $z$ and anti-analytic in $\zeta$. Suppose $\left\{c_{n}\right\}_{n=1}^{\infty}$ is a sequence of complex numbers. If

$$
\left|\sum_{n=1}^{N} \alpha_{n} c_{n}\right|^{2} \leqq \sum_{m, n=1}^{N} \alpha_{m} \bar{\alpha}_{n} K\left(z_{m}, z_{n}\right)
$$

for every finite collection of complex numbers $\alpha_{1}, \cdots, \alpha_{N}$, then there exists a unique analytic function $f$ in $D$ such that $f\left(z_{n}\right)=c_{n}$ for $n=$ $1,2, \cdots$ Furthermore,

$$
\left|\sum_{n=1}^{N} \alpha_{n} f\left(z_{n}^{\prime}\right)\right|^{2} \leqq \sum_{m, n=1}^{N} \alpha_{m} \bar{\alpha}_{n} K\left(z_{m}^{\prime}, z_{n}^{\prime}\right)
$$

holds for every finite collection of complex numbers $\alpha_{1}, \cdots, \alpha_{N}$ and any sequence $\left\{z_{n}^{\prime}\right\}$ of points.

Proof. Let $D_{1}$ be a relatively compact subdomain of $D$, containing an accumulation point of the sequence $\left\{\boldsymbol{z}_{n}\right\}$. Consider the Hilbert space $X=L_{a}^{2}\left(D_{1}\right)$ of square integrable analytic functions on $D_{1}$. Then the point evaluation functionals $x_{n}^{*}: g \rightarrow g\left(z_{n}\right)$, for $z_{n} \in D_{1}$, 
are a complete set in $X^{*}$. Define $T: X^{*} \rightarrow X$ by

$$
\left(T x^{*}\right)(z)=\int_{D_{1}} K(z, \zeta) \phi(\zeta) d m_{\zeta}
$$

where $\phi \in L_{a}^{2}\left(D_{1}\right)$ represents $x^{*}$ and $m$ is area measure. Then $T$ is a bounded conjugate-linear operator and $x_{m}^{*}\left(T x_{n}^{*}\right)=K\left(z_{m}, z_{n}\right)$. By Corollary 2.2 (or Corollary 2.1) a unique $f \in L_{a}^{2}\left(D_{1}\right)$ exists such that $f\left(z_{n}\right)=c_{n}$ for all $z_{n} \in D_{1}$. Furthermore, by choosing $x^{*}: g \rightarrow \alpha_{1} g\left(z_{1}^{\prime}\right)+$ $\cdots+\alpha_{N} g\left(z_{N}^{\prime}\right)$ in part (ii) of the corollary, we obtain (6) whenever the points $z_{1}^{\prime}, \cdots, z_{N}^{\prime}$ belong to $D_{1}$. The theorem now follows from exhausting $D$ by such subdomains $D_{1}$.

4. Some generalizations. In our proof of FitzGerald's theorem the particular choice of functionals and the complex plane were inessential. A similar proof yields the following generalization for $H(D)$, the space of analytic functions on a domain $D$ in $C^{n}$, with the topology of locally uniform convergence.

First, we need some notation: If $g$ is anti-analytic in $D$ and $x^{*} \in H^{*}(D)$, define $\bar{x}^{*} g=\overline{x^{*}(\bar{g})}$. In addition, if $K(z, \zeta)$ is defined in $D \times D$, analytic in $z$ and anti-analytic in $\zeta$, and $x^{*}, y^{*} \in H^{*}(D)$, we define $x^{*} \bar{y}^{*} K=x_{(z)}^{*}\left(\bar{y}_{(\zeta)}^{*} K(z, \zeta)\right)$. In particular, we write $\left|x^{*}\right|^{2}=x^{*} \bar{x}^{*}$. Furthermore, $K$ is a positive kernel if $\left|x^{*}\right|^{2} K \geqq 0$ for every $x^{*} \epsilon$ $H^{*}(D)$.

THEOREM 4.1. Let $D$ be a domain in $C^{n}$ and $K(z, \zeta)$ a positive kernel on $D \times D$ that is analytic in $z$ and anti-analytic in $\zeta$. Suppose that $\left\{x_{i}^{*}\right\}_{i \in I}$ is a set of functionals in $H^{*}(D)$ and that $\left\{c_{i}\right\}_{i \in I}$ is a corresponding set of complex numbers. If

$$
\left|\sum_{k=1}^{N} \alpha_{i_{k}} c_{i_{k}}\right|^{2} \leqq \sum_{k, n=1}^{N} \alpha_{i_{k}} \bar{\alpha}_{i_{k}} x_{i_{k}}^{*} \bar{x}_{i_{k}}^{*} K
$$

for every finite collection of complex numbers $\alpha_{i_{1}}, \cdots, \alpha_{i_{N}}$, then there exists a function $f \in H(D)$ such that $x_{i}^{*}(f)=c_{i}$ for all $i \in I$. Furthermore, we have

$$
\left|x^{*}(f)\right|^{2} \leqq\left|x^{*}\right|^{2} K
$$

for all $x^{*} \in H^{*}(D)$.

Proof. Let $\left\{D_{m}\right\}$ be an exhaustion of $D$ by relatively compact subdomains. Each functional $x_{i}^{*}$ may be represented by a complex measure with compact support in $D$. Let $I_{m}$ be the subset of $I$ for which the support of $x_{i}^{*}$ is in $D_{m}$. As before, let $L_{a}^{2}\left(D_{m}\right)$ be the Hilbert space of square integrable analytic functions on $D_{m}$. Since 
the topology of $L_{a}^{2}\left(D_{m}\right)$ implies uniform convergence on each compact subset of $D_{m}$, the representing measure for $x_{i}^{*}$ may be used to extend $x_{i}^{*}$ to $L_{a}^{2}\left(D_{m}\right)$ for each $i \in I_{m}$.

Define $T_{m}: L_{a}^{2}\left(D_{m}\right)^{*} \rightarrow L_{a}^{2}\left(D_{m}\right)$ by $\left(T_{m} x^{*}\right)(z)=\bar{x}_{(\xi)}^{*}\left(\left.K(z, \zeta)\right|_{D_{m} \times D_{m}}\right)$. Then $T_{m}$ is continuous, conjugate-linear, and

$$
\left\|T_{m}\right\|^{2}=\int_{D_{m} \times D_{m}}|K(z, \zeta)|^{2} d m_{z} d m_{\zeta} .
$$

It is even positive since point evaluation functionals form complete sets in $H^{*}(D)$ and $L_{a}^{2}\left(D_{m}\right)^{*}$. By Corollary 2.1 there exists a function $f_{m} \in L_{a}^{2}\left(D_{m}\right)$ such that $x_{i}^{*}\left(f_{m}\right)=c_{i}$ for each $i \in I_{m}$ and

$$
\left|x^{*}\left(f_{m}\right)\right|^{2} \leqq\left.\left|x^{*}\right|{ }^{2} K\right|_{D_{m} \times D_{m}} \text { for all } x^{*} \in L_{u}^{2}\left(D_{m}\right)^{*} \text {. }
$$

In particular, for the point evaluation functionals we have

$$
\left|f_{m}(z)\right|^{2} \leqq K(z, z) \text { for } z \in D_{m} \text {. }
$$

Therefore $\left\{f_{m}\right\}$ forms a normal family, and the limit $f$ of any convergent subsequence interpolates: $x_{i}^{*}(f)=c_{i}$ for all $i \in I$. Furthermore, since (9) holds for each finite linear combination of point evaluations if $m$ is sufficiently large, then (8) holds for such functionals. Such functionals are dense in $H^{*}(D)$; hences (8) holds for all $x^{*} \in H^{*}(D)$.

REMARKS. 1. In case $\left\{x_{i}^{*}\right\}_{i \in I}$ is a complete set of functionals, then (7) implies that $K$ is a positive kernel. This occurred in FitzGerald's theorem; however, we do not need to assume that the set of functionals is complete.

2. If the kernel $K(z, \zeta)$ of Theorem 4.1 is already square integrable over $D \times D$, then the exhaustion is not needed in the proof. As a consequence, we may conclude that there exists an interpolating function belonging to $L_{a}^{2}(D)$. Unfortunately, the Bergman kernel does not have this property. However, we shall see in Theorem 5.1 that it also produces interpolating functions in $L_{a}^{2}(D)$.

3. In case $D=C^{n}$, Theorem 4.1 gives sufficient conditions for existence of interpolating entire functions of several variables.

4. In Theorem 4.1 the index set $I$ may be uncountable. It therefore gives sufficient conditions for analytic continuation of functions defined on subsets of $D$.

5. Some obvious choices for the set of functionals are the 
following:

EXAMPLE 4.1. If $\left\{z_{i}\right\}_{i \in I}$ is a set of points in $D \subset C^{n}$, define $x_{i}^{*}(g)=g\left(z_{i}\right)$. Then (7) takes the form

$$
\left|\sum_{k=1}^{N} \alpha_{i_{k}} c_{i_{k}}\right|^{2} \leqq \sum_{k, \kappa=1}^{N} \alpha_{i_{k}} \bar{\alpha}_{i_{\kappa}} K\left(z_{i_{k}}, z_{i_{\kappa}}\right)
$$

If, for example, there is a point $\widehat{z} \in D$ such that each coordinate of $\hat{z}$ is an accumulation point of corresponding coordinates of the set $\left\{z_{i}\right\}_{i \in I}$, then the set $\left\{x_{i}^{*}\right\}_{i \in I}$ is complete and the interpolating function is unique.

EXAMPLE 4.2. If $I$ is a set of multi-indices $i=\left(m_{1}, \cdots, m_{n}\right)$ and $z_{0} \in D \subset C^{n}$ is fixed, define $x_{i}^{*}(g)=\left(\partial^{i} g / \partial z^{i}\right)\left(z_{0}\right)$. Then (7) takes the form

$$
\left|\sum_{k=1}^{N} \alpha_{i_{k}} c_{i_{k}}\right|^{2} \leqq\left.\sum_{k, \kappa=1}^{N} \alpha_{i_{k}} \bar{\alpha}_{i_{\kappa}} \frac{\partial^{i_{k}} \partial^{i_{\kappa}}}{\partial z^{i_{k}} \partial \bar{\zeta}^{i_{\kappa}}} K(z, \zeta)\right|_{z=\zeta=z_{0}} .
$$

If $I$ contains all multi-indices, then the set $\left\{x_{i}^{*}\right\}_{i \in I}$ is complete and the interpolating function is again unique.

There are many other possible choices for functionals. For example, one can use point evaluations of the function and its derivatives at various points, their linear combinations, or integrals over compact sets.

The proof of Theorem 4.1 applies equally well to the space $h(D)$ of harmonic functions on a domain $D \subset \boldsymbol{R}^{n}$, with the topology of locally uniform convergence. We simply state it:

THEOREM 4.1'. Let $D$ be a domain in $\boldsymbol{R}^{n}$ and $K(s, t)$ a continuous function on $D \times D$, that is harmonic in each variable. Assume that $K$ is a positive kernel in the sense that $\left(x^{*}\right)^{2} K=x_{(s)}^{*}\left(x_{(t)}^{*} K(s, t)\right) \geqq 0$ for every $x^{*} \in h^{*}(D)$. Suppose that $\left\{x_{i}^{*}\right\}_{i \in I}$ is a set of functionals in $h^{*}(D)$ and that $\left\{c_{i}\right\}_{i \in I}$ is a corresponding set of real numbers. If

$$
\left|\sum_{k=1}^{N} \alpha_{i_{k}} c_{i_{k}}\right|^{2} \leqq \sum_{k, k=1}^{N} \alpha_{i_{k}} \alpha_{i_{k}} x_{i_{k}}^{*} x_{i_{k}}^{*} K
$$

for every finite collection of real numbers $\alpha_{i_{1}}, \cdots, \alpha_{i_{N}}$, then there exists a function $u \in h(D)$ such that $x_{i}^{*}(u)=c_{i}$ for all $i \in I$. Furthermore, we have $\left|x^{*}(u)\right|^{2} \leqq\left(x^{*}\right)^{2} K$ for all $x^{*} \in h^{*}(D)$.

REMARKS. Just as in Example 4.1 we may choose point evaluations for the set $\left\{x_{i}^{*}\right\}_{i \in I}$ of functionals and then (10) assumes an analogous form. This set of functionals will be complete and $u$ will 
be unique if, for example, $D \subset \boldsymbol{R}^{2}$ and there are infinitely many points on some analytic Jordan curve in $D$ whose interior is also in $D$.

If, as in Example 4.2, we specify certain partial derivatives at a fixed point $t_{0} \in D$, then (10) will assume a corresponding form. Furthermore, $u$ will be unique if we interpolate all its partial derivatives at $t_{0}$.

Another proof of Theorems 4.1 and $4.1^{\prime}$ could be based on the fact that Montel spaces, such as $H(D)$ and $h(D)$, are reflexive. In this case one can apply Corollary 2.1 directly after showing that the conjugate-linear operator $T x^{*}=\bar{x}_{\xi}^{*} K$ is continuous.

5. Reproducing kernels. Let $X$ be a Hilbert space with a reproducing kernel. That is, $X$ is a space of functions on a set $D$. In addition, there is a kernel function $K(z, \zeta)$ on $D \times D$ such that

(a) $\overline{K(z, \cdot)} \in X$ for each $z \in D$, and

(b) $x(z)=(x, \overline{K(z, \cdot)})$ for each $x \in X$ and $z \in D$.

Since $X$ is a Hilbert space, the natural identification of $X^{*}$ with $X$ defines a positive, bounded, conjugate-linear operator $T$. It has the property that $x^{*}\left(T y^{*}\right)=x^{*} \bar{y}^{*} K$. Thus Corollary 2.2 implies the following.

THEOREM 5.1 (Duren-Williams). Let $X$ be a Hilbert space with a reproducing kernel $K$. Suppose that $\left\{x_{i}^{*}\right\}_{i \in I}$ is a set of functionals in $X^{*}$ and that $\left\{c_{i}\right\}_{i \in I}$ is a corresponding set of scalars. Then there exists an $x \in X$ such that

(i ) $x_{i}^{*}(x)=c_{i}$ for all $i \in I$,

(ii) $\left|x^{*}(x)\right|^{2} \leqq\left|x^{*}\right|{ }^{2} K$ for all $x^{*} \in X^{*}$, and

(iii) $\|x\| \leqq 1$,

if and only if

$$
\left|\sum_{k=1}^{N} \alpha_{i_{k}} c_{i_{k}}\right|^{2} \leqq \sum_{k, \kappa=1}^{N} \alpha_{i_{k}} \bar{\alpha}_{i_{k}} x_{i_{k}}^{*} \bar{x}_{i_{k}}^{*} K
$$

for every finite collection of scalars $\alpha_{i_{1}}, \cdots, \alpha_{i_{N}}$.

REMaRKs. This theorem was proved by P. L. Duren and D. L. Williams in [1, Thm. 2]. They stated it for a family $\left\{x_{i}^{*}\right\}_{i \in I}$ of point evaluation functionals, but their argument carries over in the present form. They obtained it as an application of a very useful, general interpolation theorem for function spaces, which in turn is based on the proof of Theorem 2.1.

Since each positive kernel generates a proper functional inner product space for which it is the reproducing kernel, a proof of Theorems 4.1 and $4.1^{\prime}$ could be based on Theorem 5.1. In this 
framework one needs to identify points in the proper functional completion with analytic or harmonic functions when the kernel has these properties.

We note a few examples of interesting kernels:

EXAMPLe 5.1. Let $D$ be a domain in $C^{n}$. A reproducing kernel $K$ for $L_{a}^{2}(D)$ is called a Bergman kernel. For example, if $D=D^{n}$ is the unit polydisk in $\boldsymbol{C}^{n}$, then the Bergman kernel is

$$
K(z, \zeta)=\pi^{-n} \prod_{k=1}^{n}\left(1-z^{(k)} \overline{\zeta^{(k)}}\right)^{-2}
$$

where $z=\left(z^{(1)}, \cdots, z^{(n)}\right)$ and $\zeta=\left(\zeta^{(1)}, \cdots, \zeta^{(n)}\right)$, and can be used in Theorem 5.1. In addition, $2 \operatorname{Re} K(z, \zeta)-1$ is the reproducing kernel for $L_{p h}^{2}\left(D^{n}\right)$, the space of square integrable pluriharmonic functions, to which Theorem 5.1 applies.

EXAMPLE 5.2. Let $H^{2}\left(\boldsymbol{D}^{n}\right)$ be the Hardy space of analytic functions $g$ in the polydisk $\boldsymbol{D}^{n}$ with the property that

$$
\|g\|^{2}=\limsup _{r \rightarrow 1} \int_{0}^{2 \pi} \cdots \int_{0}^{2 \pi}\left|f\left(r e^{i \theta_{1}}, \cdots, r e^{i \theta_{n}}\right)\right|^{2} d \theta_{1} \cdots d \theta_{n}
$$

is finite. Its reproducing kernel is

$$
K(z, \zeta)=(2 \pi)^{-n} \prod_{k=1}^{n}\left(1-z^{(k) \overline{\zeta^{(k)}}}\right)^{-1}
$$

and is called the Szegö kernel. In addition, $2 \operatorname{Re} K(z, \zeta)-1$ is a reproducing kernel for the Hardy space $h_{p h}^{2}\left(D^{n}\right)$ of pluriharmonic functions. Theorem 5.1 applies in both cases.

One easily gives many additional examples. We have mentioned these to suggest the wide applicability of Theorem 5.1.

Since Corollary 2.2 applies already to reflexive Banach spaces, one may produce interpolating functions in the spaces $L_{a}^{p}, L_{p h}^{p}, H^{p}$, $h_{p h}^{p}$ of the polydisk for $1<p \leqq 2$ for interpolating functionals in the corresponding conjugate spaces. In these cases the reproducing kernels of the examples above may be used.

For further information we refer the reader to Duren and Williams [1].

6. Interpolation and continuation. The following theorem is a generalization of a result of FitzGerald [2, Thm. 1.2], but the proof is quite different.

THEOREM 6.1. Let $D_{1}$ be a domain in $C^{m}$ and $D_{2}$ a domain in 
$C^{n}$. For $\nu=1,2$ let $K_{\nu}(z, \zeta)$ be functions on $D_{\nu} \times D_{\nu}$ that are analytic in $z$ and anti-analytic in $\zeta$. Suppose that $\left\{x_{i}^{*}\right\}_{i \in I}$ and $\left\{y_{j}^{*}\right\}_{j \in J}$ are complete sets of functionals in $H^{*}\left(D_{1}\right)$ and $H^{*}\left(D_{2}\right)$, respectively, and that $\left\{c_{i j}\right\}_{i \in I, j \in J}$ is a set of complex numbers. If

$$
\left|\sum_{k, \kappa=1}^{N_{1}, N_{2}} \alpha_{i_{k}} \beta_{j_{\kappa}} c_{i_{k} j_{\kappa}}\right|^{2} \leqq\left[\sum_{k, \kappa=1}^{N_{1}} \alpha_{i_{k}} \bar{\alpha}_{i_{\kappa}} x_{i_{k}}^{*} \bar{x}_{i_{\kappa}}^{*} K_{1}\right]\left[\sum_{k, \kappa=1}^{N_{2}} \beta_{j_{k}} \bar{\beta}_{j_{\kappa}} y_{j_{k}}^{*} \bar{y}_{j_{\kappa}}^{*} K_{2}\right]
$$

for all finite collections of complex numbers $\alpha_{i_{1}}, \cdots, \alpha_{i_{N 1}}$ and $\beta_{j_{1}}, \cdots, \beta_{j_{N 2}}$, then there exists a unique function $V \in H\left(D_{1} \times D_{2}\right)$ such that $x_{i}^{*} y_{j}^{*} V=c_{i j}$ for every $i \in I$ and $j \in J$. Furthermore, we have

$$
\left|x^{*} y^{*} V\right|^{2} \leqq\left[\left|x^{*}\right|{ }^{2} K_{1}\right]\left[\left|y^{*}\right|^{2} K_{2}\right]
$$

for all $x^{*} \in H^{*}\left(D_{1}\right)$ and $y^{*} \in H^{*}\left(D_{2}\right)$.

Proof. Choose $N_{2}=1$ in (11). Then we have

$$
\left|\sum_{k=1}^{N_{1}} \alpha_{i_{k}} c_{i_{k} j}\right|^{2} \leqq\left[\sum_{k, k=1}^{N_{1}} \alpha_{i_{k}} \bar{\alpha}_{i_{\kappa}} x_{i_{k}}^{*} \bar{x}_{i_{\kappa}}^{*} K_{1}\right]\left[\left|y_{j}^{*}\right|^{2} K_{2}\right]
$$

for all finite collections $\alpha_{i_{1}}, \cdots, \alpha_{i_{N_{1}}}$. By Theorem 4.1 there exists an $f_{j} \in H\left(D_{1}\right)$ such that $x_{i}^{*}\left(f_{j}\right)=c_{i j}$ for all $i \in I$ and also

$$
\left|x^{*}\left(f_{j}\right)\right|^{2} \leqq\left[\left|x^{*}\right|{ }^{2} K_{1}\right]\left[\left|y_{j}^{*}\right|^{2} K_{2}\right]
$$

for every $x^{*} \in H^{*}\left(D_{1}\right)$.

Let $C_{i}=\sum_{\kappa=1}^{N_{2}} \beta_{j_{\kappa}} c_{i j_{\kappa}}$ for a fixed collection $\beta_{j_{1}}, \cdots, \beta_{j_{N_{2}}}$ of complex numbers. Then (11) implies

$$
\left|\sum_{k=1}^{N_{1}} \alpha_{i_{k}} C_{i_{k}}\right|^{2} \leqq b \sum_{k, \kappa=1}^{N_{1}} \alpha_{i_{k}} \bar{\alpha}_{i_{\kappa}} x_{i_{k}}^{*} \bar{x}_{i_{\kappa}}^{*} K_{1}
$$

where $b=\sum_{k, \kappa=1}^{N_{2}} \beta_{j_{k}} \bar{\beta}_{j_{\kappa}} y_{j_{k}}^{*} \bar{y}_{j_{k}}^{*} K_{2}$. By Theorem 4.1 there exists an $F \in H\left(D_{1}\right)$ such that $x_{i}^{*}(F)=C_{i}$ for all $i \in I$ and, moreover,

$$
\left|x^{*}(F)\right|^{2} \leqq b\left|x^{*}\right|^{2} K_{1} \quad \text { for every } \quad x^{*} \in H^{*}\left(D_{1}\right) .
$$

Since $\sum_{k=1}^{N_{2}} \beta_{j_{k}} f_{j_{k}}$ also interpolates the set $\left\{C_{i}\right\}_{i \in I}$ and the family $\left\{x_{i}^{*}\right\}_{i \in I}$ is complete, we conclude that $F=\sum_{\kappa=1}^{N_{2}} \beta_{j_{k}} f_{j_{k}}$. Therefore, taking $x^{*}$ in (13) to be point evaluation at $z$, we have

$$
\left|\sum_{\kappa=1}^{N_{2}} \beta_{j_{k}} f_{j_{k}}(z)\right|^{2} \leqq K_{1}(z, z) \sum_{k, k=1}^{N_{2}} \beta_{j_{k}} \bar{\beta}_{j_{\kappa}} y_{j_{k}}^{*} \bar{y}_{j_{\kappa}}^{*} K_{2} \text {. }
$$

Holding $z$ fixed, inequality (14) is valid for every finite collection $\beta j_{1}, \cdots, \beta j_{N_{2}}$. Again by Theorem 4.1 there exists a function $G_{z} \in$ $H\left(D_{2}\right)$ such that $y_{j}^{*}\left(G_{z}\right)=f_{j}(z)$ for every $j \in J$ and also

$$
\left|y^{*}\left(G_{z}\right)\right|^{2} \leqq K_{1}(z, z)\left|y^{*}\right|{ }^{2} K_{2}
$$


for all $y^{*} \in H^{*}\left(D_{2}\right)$. Since the family $\left\{y_{j}^{*}\right\}_{j \in J}$ is complete, we may find finite linear combinations $y_{\nu}^{*}=\sum_{\mu=1}^{M_{\nu}} \gamma_{j_{\mu \nu}} y_{j_{\mu \nu}}^{*}$ that converge to the point evaluation functional at a fixed point $\zeta_{0}$. In this case (15) implies a uniform bound on compact subsets of $D_{1}$ for the analytic functions $y_{\nu}^{*}\left(G_{z}\right)=\sum_{\mu=1}^{M_{\nu}} \gamma_{j_{\mu \nu}} f_{j_{\mu \nu}}(z)$ of $z$. Consequently, their limit $G_{z}\left(\zeta_{0}\right)$ is analytic in $z$.

Define $V(z, \zeta)=G_{z}(\zeta)$. Then $V$ is analytic in each variable and so belongs to $H\left(D_{1} \times D_{2}\right)$. Furthermore, $x_{i}^{*} y_{j}^{*} V=x_{i}^{*}\left(f_{j}\right)=c_{i j}$ for all $i \in I$ and $j \in J$. Finally, (12) follows from (11) since the families $\left\{x_{\imath}^{*}\right\}_{i \in I}$ and $\left\{y_{j}^{*}\right\}_{j \in J}$ are complete. $V$ is unique for the same reason.

Remarks. FitzGerald's theorem [2, Thm. 1.2] is the special case $m=n=1$ where the sets of functionals are sequences of point evaluations, both having an interior accumulation point. As in the examples following Theorem 4.1, there are many other possible choices for functionals. Furthermore, the index sets $I$ and $J$ do not need to be denumerable.

The following corollaries are fairly direct consequences of Theorem 6.1. We omit their proofs since they are analogous to those of FitzGerald [2, Theorems 1.3 and 1.4].

Corollary 6.1. Let $D$ be a domain in $C^{n}$ and $K(z, \zeta)$ a function on $D \times D$, that is analytic in $z$ and anti-analytic in $\zeta$. Suppose that $\left\{x_{i}^{*}\right\}_{i \in I}$ is a complete set of functionals in $H^{*}(D)$ and that $\left\{c_{i j}\right\}_{i, j \in I}$ is a symmetric $\left(c_{i j}=c_{j i}\right)$ set of complex numbers. If

$$
\left|\sum_{k, k=1}^{N} \alpha_{i_{k}} \alpha_{i_{\kappa}} c_{i_{k} i_{\kappa}}\right| \leqq \sum_{k, \kappa=1}^{N} \alpha_{i_{k}} \bar{\alpha}_{i_{\kappa}} x_{i_{k}}^{*} \bar{x}_{i_{\kappa}}^{*} K
$$

for every finite collection of complex numbers $\alpha_{i_{1}}, \cdots, \alpha_{i_{N}}$, then there exists a unique function $V \in H(D \times D)$ such that $x_{i}^{*} x_{j}^{*} V=c_{i j}$ for all $i, j \in I . \quad$ Furthermore, $\quad V$ is symmetric $(V(z, \zeta)=V(\zeta, z))$ and $\left|\left(x^{*}\right)^{2} V\right| \leqq\left|x^{*}\right|^{2} K$ for every $x^{*} \in H^{*}(D)$.

Corollary 6.2. Let $D_{1}$ and $D_{2}$ be domains in $C^{n}$ with nonempty intersection and $K(z, \zeta)$ a function defined on $\left(D_{1} \times D_{1}\right) \cup\left(D_{2} \times D_{2}\right)$, that is analytic in $z$ and anti-analytic in $\zeta$. Suppose that $\left\{x_{i}^{*}\right\}_{i \in I}$ is a complete set of functionals in $H^{*}\left(D_{1} \cap D_{2}\right)$. If

$$
\sum_{k, k=1}^{N} \alpha_{i_{k}} \bar{\alpha}_{i_{\kappa}} x_{i_{k}}^{*} \bar{x}_{i_{\kappa}}^{*} K \geqq 0
$$

for every finite collection of complex numbers $\alpha_{i_{1}}, \cdots, \alpha_{i_{N}}$, then $K(z, \zeta)$ can be continued to a positive kernel on $\left(D_{1} \cup D_{2}\right) \times\left(D_{1} \cup D_{2}\right)$ that is analytic in $z$ and anti-analytic in $\zeta$. 
By using Theorem 4.1' in place of Theorem 4.1 one obtains the following parallel results for harmonic interpolation and continuation.

Theorem 6.1'. Let $D_{1}$ be a domain in $\boldsymbol{R}^{m}$ and $D_{2}$ a domain in $\boldsymbol{R}^{n}$. For $\nu=1,2$ let $K_{\nu}(s, t)$ be continuous functions on $D_{\nu} \times D_{\nu}$, that are harmonic in each variable. Suppose that $\left\{x_{i}^{*}\right\}_{i \in I}$ and $\left\{y_{j}^{*}\right\}_{j \in J}$ are complete sets of functionals in $h^{*}\left(D_{1}\right)$ and $h^{*}\left(D_{2}\right)$, respectively, and that $\left\{c_{i j}\right\}_{i \in I, j \in J}$ is a set of real numbers. If

$$
\left|\sum_{k, \kappa=1}^{N_{1}, N_{2}} \alpha_{i_{k}} \beta_{j_{\kappa}} c_{i_{k} j_{\kappa}}\right|^{2} \leqq\left[\sum_{k, \kappa=1}^{N_{1}} \alpha_{i_{k}} \alpha_{i_{\kappa}} x_{i_{k}}^{*} x_{i_{\kappa}}^{*} K_{1}\right]\left[\sum_{k, \kappa=1}^{N_{2}} \beta_{j_{k}} \beta_{j_{\kappa}} y_{j_{k}}^{*} y_{j_{\kappa}}^{*} K_{2}\right]
$$

for all finite collections of real numbers $\alpha_{i_{1}}, \cdots, \alpha_{i_{N_{1}}}$ and $\beta_{j_{1}}, \cdots, \beta_{N_{N_{2}}}$, then there exists a unique continuous function $V$ on $D_{1} \times D_{2}$ that is harmonic in each variable and satisfies $x_{i}^{*} y_{j}^{*} V=c_{i j}$ for every $i \in I$ and $j \in J$. Furthermore, we have $\left|x^{*} y^{*} V\right|^{2} \leqq\left[\left(x^{*}\right)^{2} K_{1}\right]\left[\left(y^{*}\right)^{2} K_{2}\right]$ for all $x^{*} \in h^{*}\left(D_{1}\right)$ and $y^{*} \in h^{*}\left(D_{2}\right)$.

Corollary 6.1'. Let $D$ be a domain in $\boldsymbol{R}^{n}$ and $K(s, t)$ a continuous function on $D \times D$ that is harmonic in each variable. Suppose that $\left\{x_{i}^{*}\right\}_{i \in I}$ is a complete set of functionals in $h^{*}(D)$ and that $\left\{c_{i j}\right\}_{i, j \in I}$ is a symmetric set of real numbers. If

$$
\left|\sum_{k, \kappa=1}^{N} \alpha_{i_{k}} \alpha_{i_{\kappa}} c_{i_{k} i_{\kappa}}\right| \leqq \sum_{k, \kappa=1}^{N} \alpha_{i_{k}} \alpha_{i_{\kappa}} x_{i_{k}}^{*} x_{i_{\kappa}}^{*} K
$$

for every finite collection of real numbers $\alpha_{i_{1}}, \cdots, \alpha_{i_{N}}$, then there exists a unique continuous function $V$ on $D \times D$ that is harmonic in each variable and satisfies $x_{i}^{*} x_{j}^{*} V=c_{i j}$ for all $i, j \in I$. Furthermore, $V$ is symmetric and $\left|\left(x^{*}\right)^{2} V\right| \leqq\left(x^{*}\right)^{2} K$ for every $x^{*} \in h^{*}(D)$.

Corollary 6.2'. Let $D_{1}$ and $D_{2}$ be domains in $\boldsymbol{R}^{n}$ with nonempty intersection and $K(s, t)$ a symmetric continuous function on $\left(D_{1} \times D_{1}\right) \cup$ $\left(D_{2} \times D_{2}\right)$ that is harmonic in each variable. Suppose that $\left\{x_{i}^{*}\right\}_{i \in I}$ is a complete set of functionals in $h^{*}\left(D_{1} \cap D_{2}\right)$. If

$$
\sum_{k, \kappa=1}^{N} \alpha_{i_{k}} \alpha_{i_{\kappa}} x_{i_{k}}^{*} x_{i_{\kappa}}^{*} K \geqq 0
$$

for every finite collection of real numbers $\alpha_{i_{1}}, \cdots, \alpha_{i_{N}}$, then $K(s, t)$ can be continued to a positive kernel on $\left(D_{1} \cup D_{2}\right) \times\left(D_{1} \cup D_{2}\right)$ that is continuous and is harmonic in each variable.

7. An application. This section contains an extension of PickNevanlinna interpolation and some remarks. 
THEOREM 7.1. Let $\left\{z_{i}\right\}_{i \in I}$ be a set of uniqueness for $H\left(D^{n}\right)$ and $\left\{w_{\imath}\right\}_{i \in I} a$ set of complex numbers. For $z$ and $\zeta$ in the polydisk $\boldsymbol{D}^{n}$ assume that a kernel $K$ has the form

$$
K(z, \zeta)=1+\sum a_{m_{1} m_{2} \cdots m_{n}}\left(z^{(1)} \overline{\zeta^{(1)}}\right)^{m_{1}}\left(z^{(2)} \overline{\zeta^{(2)}}\right)^{m_{2}} \cdots\left(z^{(n)} \overline{\zeta^{(n)}}\right)^{m_{n}}
$$

where all $a_{m_{1} m_{2} \cdots m_{n}} \geqq 0$. Then there exists an analytic function $f: \boldsymbol{D}^{n} \rightarrow \overline{\boldsymbol{D}}$ such that

(i ) $f\left(z_{i}\right)=w_{i}$ for all $i \in I$ and

(ii) $\left|x^{*}\right|^{2}([1-f(z) \overline{f(\zeta)}] K(z, \zeta)) \geqq 0$ for all $x^{*} \in H^{*}\left(D^{n}\right)$

if and only if

$$
\sum_{k, k=1}^{N} \alpha_{i_{k}} \bar{\alpha}_{i_{\kappa}}\left(1-w_{i_{k}} \bar{w}_{i_{\kappa}}\right) K\left(z_{i_{k}}, z_{i_{k}}\right) \geqq 0
$$

for all finite collections of complex numbers $\alpha_{i_{1}}, \cdots, \alpha_{i_{N}}$.

Proof. Inequality (16) is an immediate consequence of (i) and (ii) for the functional $x^{*}$ defined by $x^{*}(g)=\sum_{k=1}^{N} \alpha_{i_{k}} g\left(z_{i_{k}}\right)$.

Conversely, if (16) holds, then

$$
\begin{aligned}
\sum_{k, k=1}^{N} & \alpha_{i_{k}} \bar{\alpha}_{i_{k}} K\left(z_{i_{k}}, z_{i_{k}}\right) \geqq \sum_{k, \kappa=1}^{N} \alpha_{i_{k}} \bar{\alpha}_{i_{k}} w_{i_{k}} \bar{w}_{i_{k}} K\left(z_{i_{k}}, z_{i_{k}}\right) \\
& =\left|\sum_{k=1}^{N} \alpha_{i_{k}} w_{i_{k}}\right|^{2}+\sum a_{m_{1} \cdots m_{n}}\left|\sum_{k=1}^{N} \alpha_{i_{k}} w_{i_{k}}\left(z_{i_{k}}^{(1)}\right)^{m_{1}} \cdots\left(z_{i_{k}}^{(n)}\right)^{m_{n}}\right|^{2} \\
& \geqq\left|\sum_{k=1}^{N} \alpha_{i_{k}} w_{i_{k}}\right|^{2}
\end{aligned}
$$

for all $\alpha_{i_{1}}, \cdots, \alpha_{i_{N}} \in \boldsymbol{C}$. Since the family $\left\{x_{i}^{*}\right\}_{i \in I}$ of point evaluations at $z_{i}$ is complete, $K$ is a positive kernel and by Theorem 4.1 there exists a (unique) function $f \in H\left(D^{n}\right)$ that satisfies (i). Now (ii) follows from (16) and the completeness of $\left\{x_{i}^{*}\right\}_{i \in I}$. Finally, to verify that $f\left(D^{n}\right) \subset \bar{D}$ one inserts point evaluation functionals into (ii) and uses the positivity of $K$.

REMARKS. Note that the Bergman and Szegö kernels of Examples 5.1 and 5.2 satisfy the hypothesis of Theorem 7.1.

One easily obtains analogous versions of Theorem 7.1 for harmonic or pluriharmonic functions.

For our final remark, let us restrict our attention to the unit disk $\boldsymbol{D}$ and the kernels

$$
K(z, \zeta)=\frac{1}{(1-z \bar{\zeta})^{p}}
$$

where $p$ is a positive integer. In this case, if $\left\{z_{n}\right\}_{n=1}^{\infty}$ is a sequence 
of distinct points in $\boldsymbol{D}$, with an accumulation point in $\boldsymbol{D}$, and if

$$
\sum_{m, n=1}^{N} \alpha_{m} \bar{\alpha}_{n} \frac{1-w_{m} \bar{w}_{n}}{\left(1-z_{m} \bar{z}_{n}\right)^{p}} \geqq 0
$$

for all finite collections $\alpha_{1}, \cdots, \alpha_{N} \in C$, then Theorem 7.1 implies that there is an analytic function $f$ in $\boldsymbol{D}$ that is bounded by 1 and that satisfies $f\left(z_{n}\right)=w_{n}$ for every $n=1,2,3, \cdots$. In (17) the special case $p=1$ is, of course, the classical Pick-Nevanlinna conditions.

It is interesting to note that the conditions (17) are necessary for all $p=1,2,3, \cdots$. For $p=1$ one usually bases a proof on the Herglotz representation for analytic functions with positive real part. This proof extends easily to (17). Alternatively, one may deduce the cases $p=2,3, \cdots$ from $p=1$ by writing

$$
\frac{1-w_{m} \bar{w}_{n}}{\left(1-z_{m} \bar{z}_{n}\right)^{p}}=\frac{1-w_{m} \bar{w}_{n}}{1-z_{m} \bar{z}_{n}} \frac{1}{\left(1-z_{m} \bar{z}_{n}\right)^{p-1}} \text {. }
$$

Each factor generates a nonnegative hermitian form, and the Schur product theorem ensures that their product does also. Consequently, the conditions (17) are necessary and sufficient for every $p=$ $1,2,3, \cdots$.

\section{REFERENCES}

1. P. L. Duren and D. L. Williams, Interpolation problems in function spaces, J. Functional Analysis, 9 (1972), 75-86.

2. C. H. FitzGerald, Quadratic inequalities and analytic continuation, J. Analyse Math., 31 (1977), 19-47.

3. C. H. FitzGerald and R. A. Horn, On quadratic and bilinear forms in function theory, Proc. London Math. Soc., to appear.

4. G. Köthe, Topological Vector Spaces II, Grundlehren der math. Wiss. 237, SpringerVerlag, 1979.

Received November 3, 1980 and in revised form December 29, 1980. This work was supported in part by grants from the National Research Council (Canada) and the National Science Foundation (USA).

UNIVERSITÉ LAVAL

QUÉBEC, P. Q. G1K 7P4

AND

INDIANA UNIVERSITY

BLOOMINGTON, IN 47405 



\section{PACIFIC JOURNAL OF MATHEMATICS}

\section{EDITORS}

DONALD BABBITT (Managing Editor)

University of California

Los Angeles, California 90024

Hugo RossI

University of Utah

Salt Lake City, UT 84112

C. C. MOORE and ARTHUR Agus

University of California

Berkeley, CA 94720

\section{J. DUGUNDJI}

Department of Mathematics University of Southern California Los Angeles, California 90007

R. FinN and J. MILGRAM Stanford University Stanford, California 94305

\section{ASSOCIATE EDITORS}
R. ARNES
E. F. BeCKENBACH
B. H. Neumann
F. WOLF
K. YosHIDA

\section{SUPPORTING INSTITUTIONS}

UNIVERSITY OF ARIZONA

UNIVERSITY OF BRITISH COLUMBIA

CALIFORNIA INSTITUTE OF TECHNOLOGY

UNIVERSITY OF CALIFORNIA

MONTANA STATE UNIVERSITY

UNIVERSITY OF NEVADA, RENO

NEW MEXICO STATE UNIVERSITY

OREGON STATE UNIVERSITY
UNIVERSITY OF OREGON UNIVERSITY OF SOUTHERN CALIFORNIA STANFORD UNIVERSITY UNIVERSITY OF HAWAII UNIVERSITY OF TOKYO UNIVERSITY OF UTAH WASHINGTON STATE UNIVERSITY UNIVERSITY OF WASHINGTON 


\section{Pacific Journal of Mathematics}

Vol. 100, No. $1 \quad$ September, 1982

Charalambos D. Aliprantis, Owen Sidney Burkinshaw and M. Duhoux,

Compactness properties of abstract kernel operators $\ldots \ldots \ldots \ldots \ldots \ldots 1$

Roger C. Alperin, Locally compact groups acting on trees .............23

Robert F. Brown, Real homology of Lie group homomorphisms ......... 33

Karen Chase, Maximal groups in sandwich semigroups of binary relations . . 43

W. Wistar (William) Comfort and T. Soundararajan, Pseudocompact

group topologies and totally dense subgroups $\ldots \ldots \ldots \ldots \ldots \ldots \ldots 61$

M. Ferri and C. Gagliardi, Crystallisation moves $\ldots \ldots \ldots \ldots \ldots \ldots \ldots$

Kenneth R. Goodearl, Directly finite aleph-nought-continuous regular

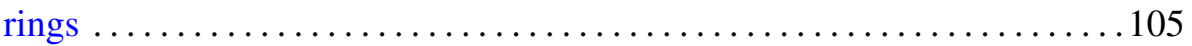

Edward Lewis Green, On the representation theory of rings in matrix

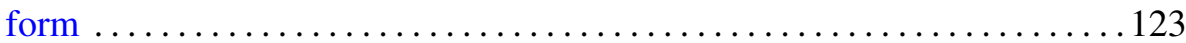

Walter Hengartner and Glenn E. Schober, Interpolation, continuation, and

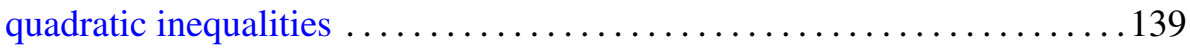

Kenneth Kunen and Haskell Paul Rosenthal, Martingale proofs of some geometrical results in Banach space theory $\ldots \ldots \ldots \ldots \ldots \ldots \ldots \ldots \ldots \ldots$

Brian William McEnnis, Shifts on indefinite inner product spaces. II . . . . . 177

Roman Pol, Note on the spaces $P(S)$ of regular probability measures whose topology is determined by countable subsets $\ldots \ldots \ldots \ldots \ldots \ldots \ldots \ldots 185$

Joan Manuel Verdera Melenchón, Finitely generated projective extensions

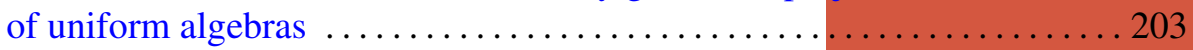

Cheng Ye You, Fixed point classes of a fiber map .................. 217 